\title{
Genetic screening
}

National Cancer Institute ( $\mathrm{NCl})$

\section{Source}

National Cancer Institute (NCI). Genetic screening.

Genetic testing designed to identify individuals in a given population who are at higher risk of having or developing a particular disorder, or carrying a gene for a particular disorder. 